\title{
A 3D, Interactive Virtual Instruction Laboratory
}

Mr. Ye Li, Univ of Illinois

A first-year graduate student at the University of Illinois.

Prof. Rizwan Uddin, University of Illinois, Urbana-Champaign

Mr. Xuefeng Zhu

Imran Haddish 


\title{
A 3D, Interactive Virtual Instruction Laboratory
}

\author{
Imran Haddish, Ye Li, Xuefeng Zhu and Rizwan-uddin \\ Department of Nuclear, Plasma, and Radiological Engineering \\ University of Illinois at Urbana-Champaign, Urbana, IL 61801 \\ haddish1@gmail.com,yeli4@illinois.edu,rizwan@illinois.edu
}

\section{Introduction}

Effective utilization of new computer technologies is essential to furthering engineering education and to encourage youth to pursue studies in STEM fields. Laboratories are a very important part of such training. Recent increase in the student population in nuclear engineering programs has put strains on laboratory resources. This increase in student population, constraints on resources and qualitative improvements in gaming technology have led researchers in the field of radiological and nuclear engineering to explore virtual, game-like models to provide the needed experience [1-3]. Though virtual lab experience may never completely replace an actual physical lab experience in educational institutions, in some ways virtual labs may provide a better experience than limited cookbook style executions in a physical lab or reactor operator training course.

We have earlier reported our initial efforts toward the development of a generic virtual and interactive laboratory environment [3]. This virtual lab presents a fully immersive learning experience. We here report the specifics of a radiation lab in which half-life and shielding experiments can be conducted, and simulation-based real-physics data can be gathered.

\section{Virtual Lab}

The primary resource for the development of a virtual lab is a game engine. Built-in features in modern game engines provide easy and quick development of the lab, as well as reduce the effort needed to develop the virtual operational procedure (user interaction step) of the lab. The development process of the virtual lab consists of three major parts: environment modeling; event scripting; and user interaction. The physics model of the experiment to be conducted also needs to be scripted. Depending on the complexity of the laboratory space, the environment modeling part can be carried out in either a 3D modeling software, or inside the game engine, such as Unity 3D. For generic items such as rooms, ladders, tables, chairs, computer monitors, etc., the game engines provide sufficient support. However, for specific items such as a Geiger counter, spectrometer, etc., models may need to be developed in a 3D modeling software. Number of custom made components increase if the virtual lab needs to appear like a real (physical) lab. Figure 1 shows a side-by-side view of a digital gamma-ray spectrometer in the lab (right) and its virtual counterpart (left). Some, and not necessarily all, of the buttons, switches and displays on these virtual models may be made "live", i.e., a user can use them to turn the equipment on or off, or set parameter values (by clicking on the object). A live data display part is programmed to display the data (from a real time simulation, experiment or pre-generated). 


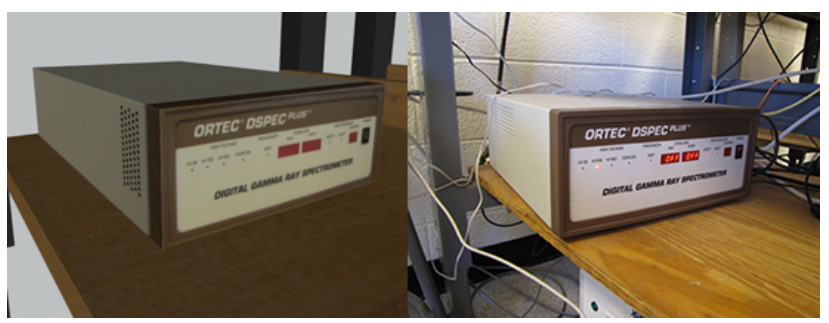

Fig. 1. A side-by-side view of a gamma-ray spectrometer in the lab (right) and its virtual counterpart (left).

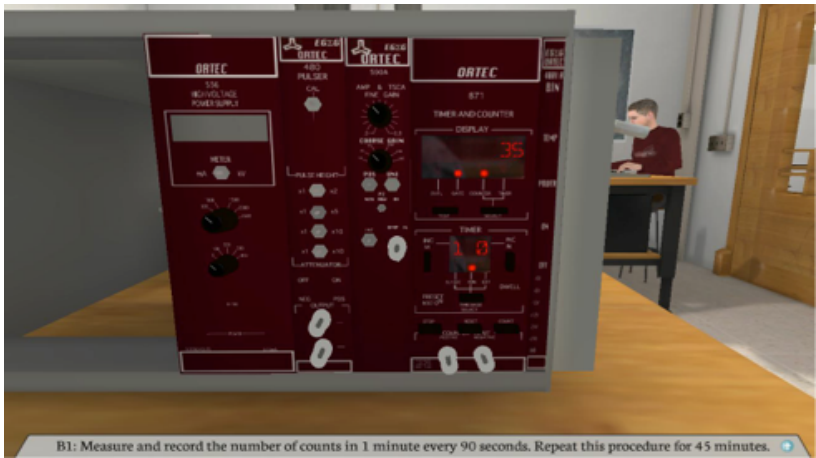

Fig. 2. Virtual model of a device showing live switches/buttons and digital displays.

In the event scripting part, the developer needs to decide how much control to give to the end user. This can vary from limited control (limited number of movable objects and control of a few switches and buttons) to as much control as they would have in real life. Figure 2 shows the model of an ORTEC power supply and a timer-plus-counter, which is used to conduct the two radiation experiments. At this point, three buttons are live. The first one is used to select the unit for the time interval. It is a toggle switch for choices between $0.1 \mathrm{~s}$ and $1 \mathrm{~min}$. The second and third live buttons control the unit and the tenth places respectively, of the time interval to be selected for the count. (If the time interval selected is 50 units while the unit switch was selected to be $0.1 \mathrm{~s}$, then the count will take place over $(0.1 \times 50=) 5$ seconds.) There is also one digital display that can show either the current count or the time lapsed during the count. The display can be toggled back and forth by clicking on a button below the digital display. The device works as it would in real life, so the student must understand how to use it. Procedures for the two virtual labs developed so far are very similar to the procedures student follow in the physical lab. Physics models have also been implemented to calculate and display data in real time that would be, for all practical purposes, indistinguishable from the data that would be gathered in the corresponding physical lab. Figure 3 shows a comparison of the physical lab and its virtual counterpart.

\section{Half-Life (Virtual) Lab}

In this experiment, physics model is programmed to calculate and display the radiation counts from a radiation source whose half-life is to be measured. Students select the unit for the time interval, and set the time interval over which the count is to be measured, and then click on the (virtual) count button to start the count. The counts are displayed, and automatically stop after the pre-set time interval is reached. Students wait for a time interval and then repeat the process, noting down the counts. Counts displayed are calculated using an exponential function $\left(e^{-\lambda t}\right)$, with the half-life of the sample radiation source. Noise is added to the calculated count rate before displaying it, to make the data more realistic (and to make it different for different runs).
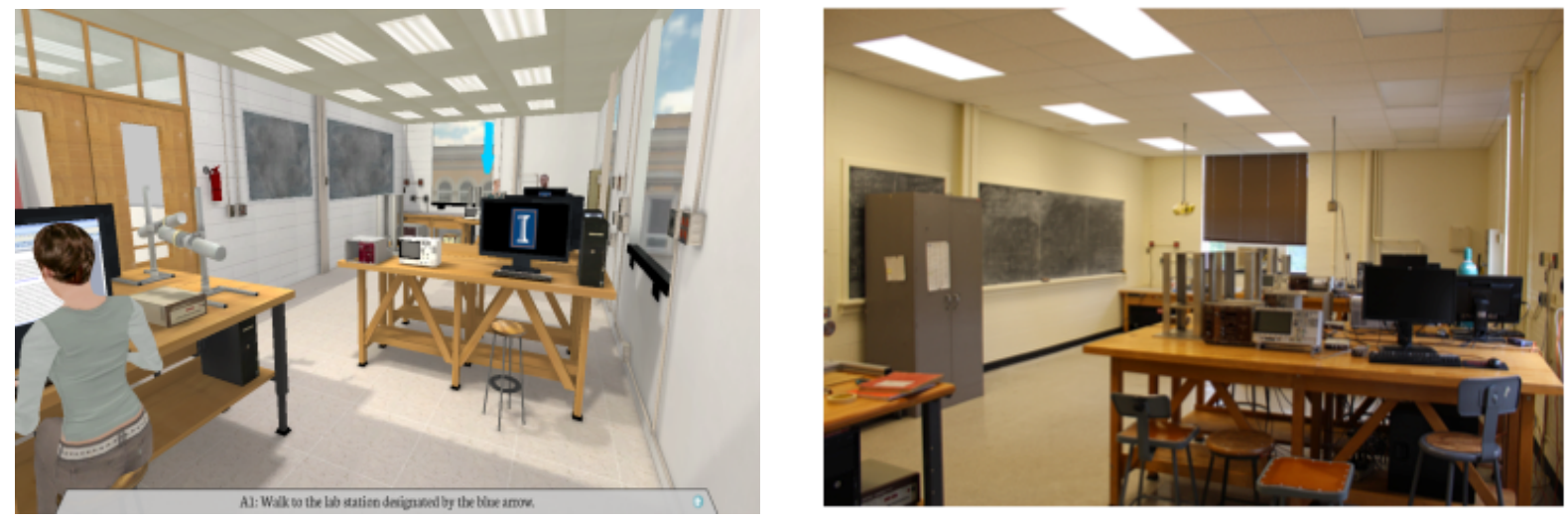

Fig. 3. A picture of the physical lab (left) and a screen shot of the virtual lab (right). 


\section{Shielding (Virtual) Lab}

In this lab, the attenuation coefficients of four different materials are to be measured. Physics model is based on the attenuation model $\left(e^{-\mu \Delta x}\right)$, where $\Delta x$ is the thickness of the shielding material. Student clicks on the shielding blocks placed on the table, which moves them next to a scale, allowing the student to measure their thickness. Next, clicking on the block moves it to the space between the radiation source and the detector. Counts can then be measured by setting the time interval and clicking on the counter button. Process is repeated for different number of shielding blocks; thus gathering data for different thicknesses. Entire process can then be repeated for blocks made of different material. Figure 4 shows the thickness measurement step. Figure 5 shows the lead shielding blocks, next to labels A, B, C, ..., for use in the shielding experiment. Figure 6 shows two of the shielding blocks placed in between the radiation source (on the left) and the detector (on the right). Collimator blocks can also be seen.

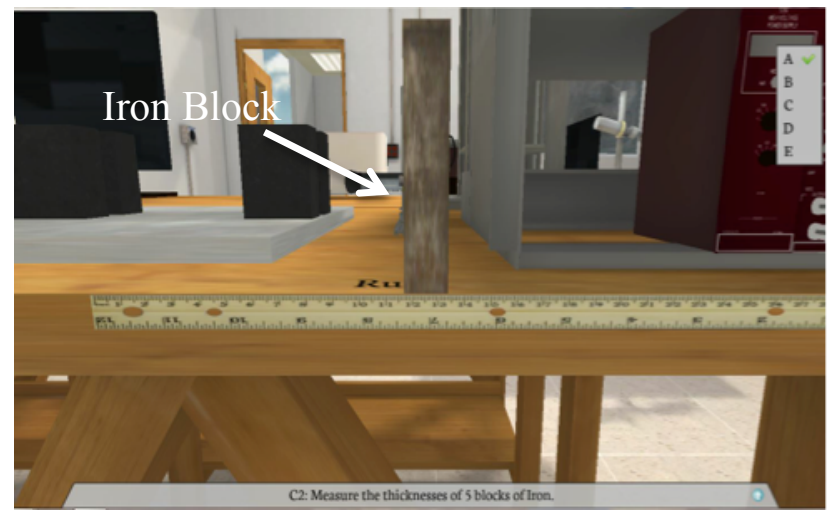

Fig. 4. Virtual lab showing the step where the student measures the thickness of the shielding block by placing it against a measuring scale

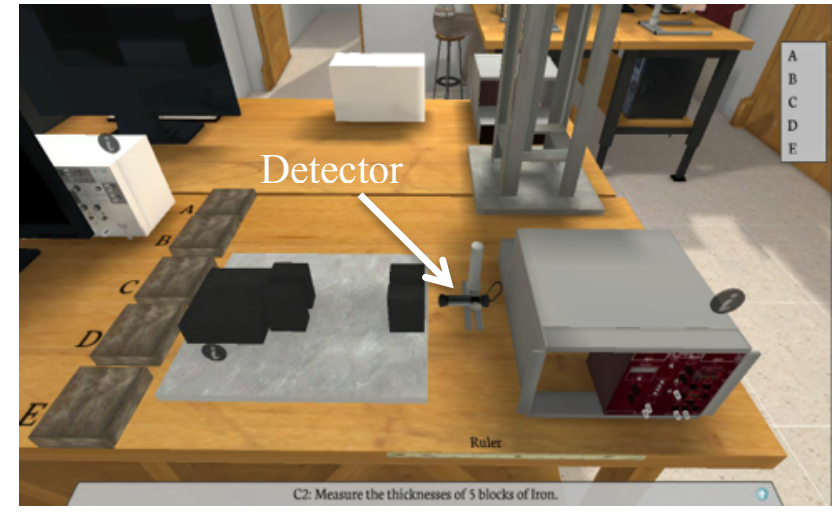

Fig. 5. Virtual lab showing the shielding blocks (labeled A, B, C ...) and the shielding experiment.

\section{Student Experience and Improvements}

The two virtual labs were used by students during the fall semesters of 2012 and 2013. Initial feedback has been very positive, though there are recommendations that are likely to further improve the student experience. Based on the recommendations from 2012, additional instructions were added to further improve the virtual laboratory experience. In addition to these improvements, a new micro sensor Leap Motion Controller (capable of tracking finger motions) - is now under calibration and will be integrated into the virtual lab to provide users with a finger-rather than mouse-based control of the lab equipment. Though we did carry out an informal study [6], we have not yet carried out a systematic study to assess the utility of virtual laboratories. However, there is a rapidly growing body of literature on the pedagogy and utility of education and training using virtual environments [4]. 


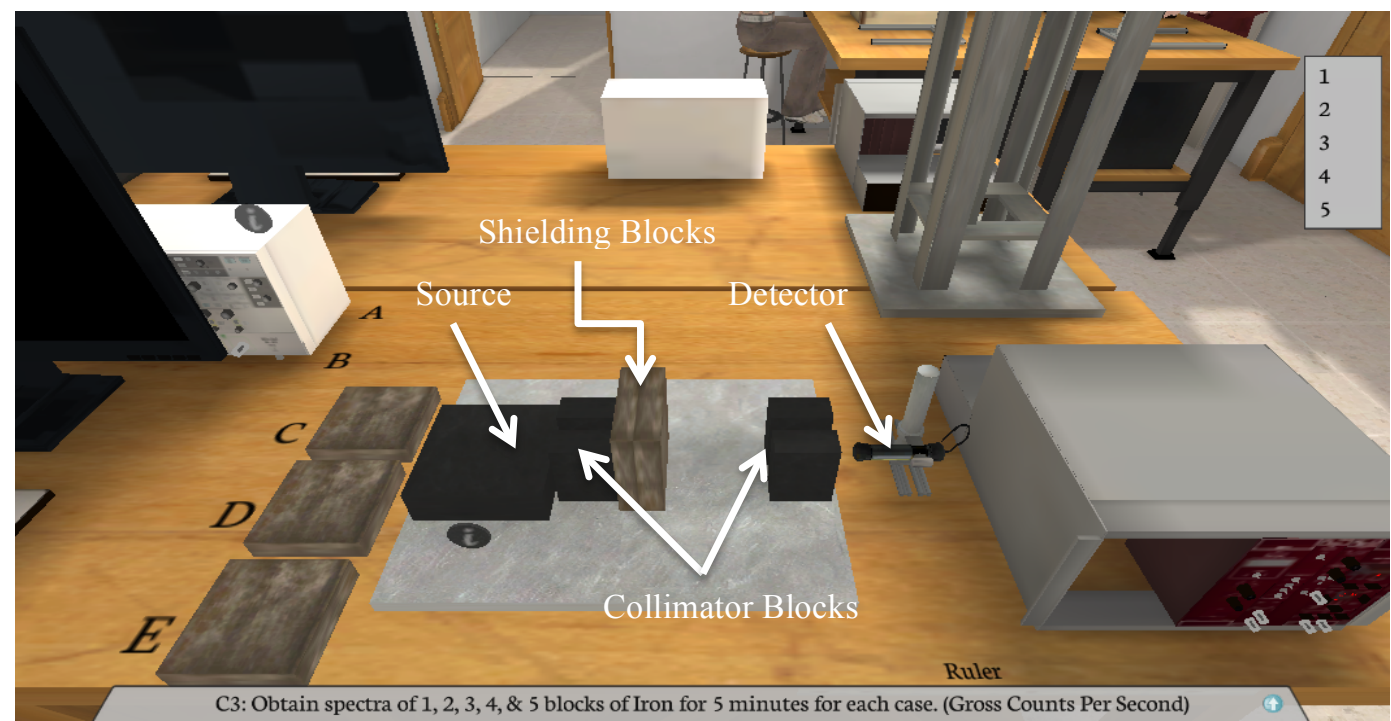

Fig. 6. Virtual model showing two blocks placed in between the source and the detector

\section{Current Efforts and Long Term Goals}

Currently, our efforts are focused on the extension of the UIUC TRIGA reactor model, that was developed earlier [5]. This model has also been developed on the Unity platform and some new interactive features have also been added [7]. The control room of the reactor model has recently been remodeled. Figure 7 shows the model of the control room, which will be used to conduct virtual experiments and to display the data in the reactor lab experiments. Figure 8 shows the live buttons of the control panel and real-time digital display capability, showing the current reactor power level. The change in slopes of the power level are due to the movements of the control rod made possible by pressing the (virtual) "UP" and "DOWN" buttons. Currently, a simple point reactor kinetics model with one delayed neutron group constitutes the physics model.

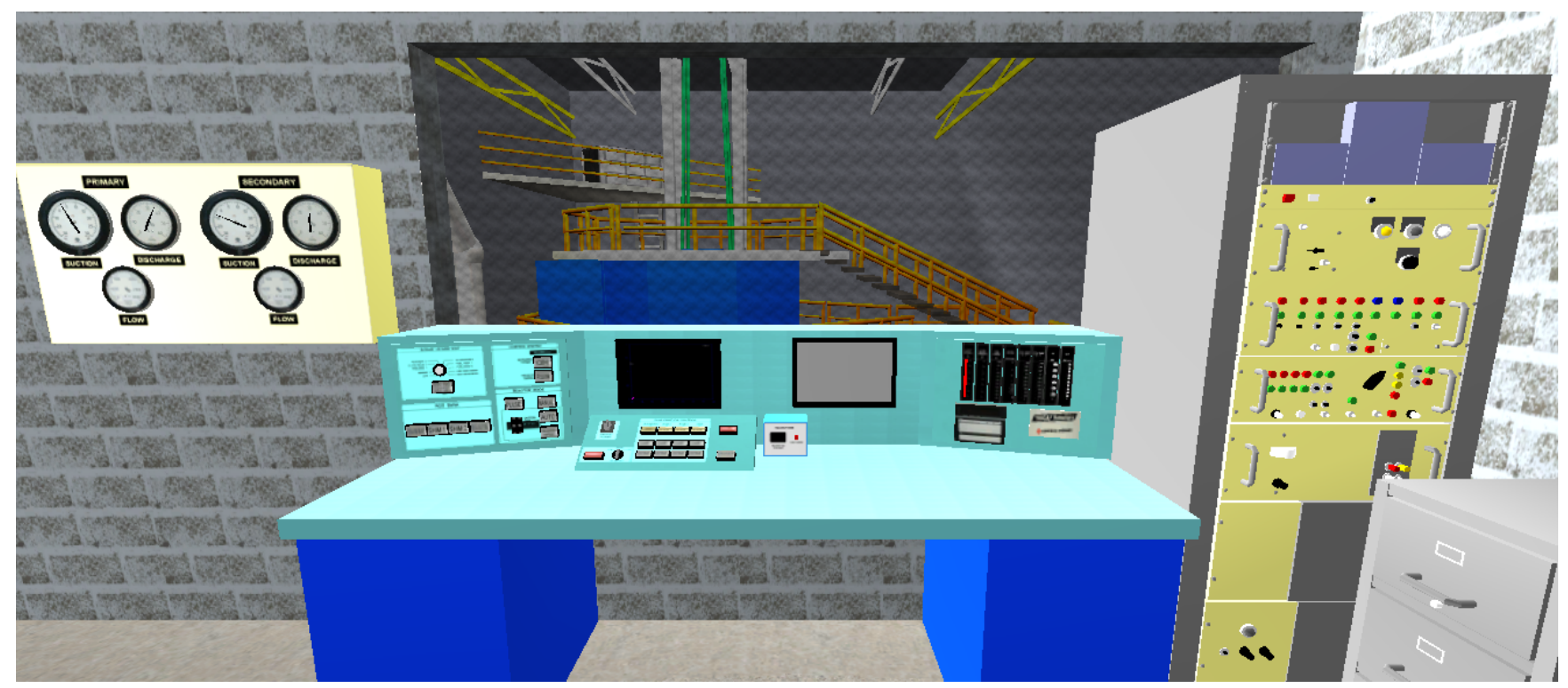

Fig. 7. The UIUC TRIGA reactor control room. This is part of the model of the complete reactor facility 

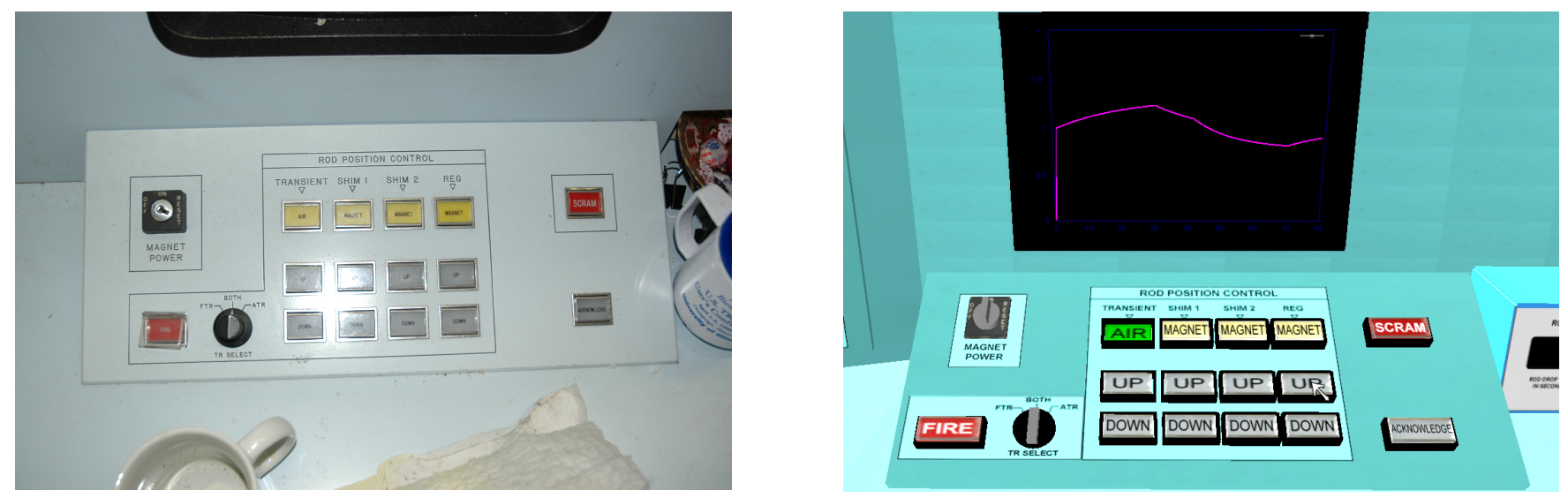

Fig. 8. A picture of one part of the UIUC TRIGA reactor control panel (left) and a screen shot of the virtual counterpart (right).

\section{References}

1. SANDERS R. L., RHODES G. S., “A Simulation Learning Approach to Training First Responders for Radiological Emergencies.” Proceedings of the 2007 Summer Computer Simulation Conference. July 15 - 17, 2007.

2. Mo'1, A.C.A., JORGE, C.A.F., COUTO, P.M., AUGUSTO, S.C., CUNHA, G.G., LANDAU, L., Virtual Environments Simulation for Dose Assessment in Nuclear Plants, Progress in Nuclear Energy 51, 382-387, 2009

3. IMRAN HADDISH, RIZWAN-UDDIN, YE LI, "FULLY INTERACTIVE VIRTUAL LABS FOR TRAINING AND EDUCATION”, Proc. ANS CONTE Meeting, Jacksonville, FL (2013).

4. R. LINDGREN, M. MOSHELL, E. HUGHES, "Virtual Environments as a tool for conceptual learning”, In K. HALE \& K. M. STANNEY (Eds.), Handbook of virtual environments: Design, implementation, and applications (2nd Edition).

5. J. DIXON, S. MARKIDIS, C.LOU, J. REYNOLDS, RIZWAN-UDDIN, “Three Dimensional, Virtual, Game-Like Environments for Education and Training", Proc, ANS, p. 97, (2007)

6. Z. KRIZ, H. WU, C.A. MORROW, C. RYTYCH, L. CONLEY, R. PROCHASKA, C. VASQUEZ AND RIZWAN-UDDIN, "An Assessment of a Game-Like 3-D Model for Training at NPPs", Tran. ANS, p. 102, (2010)

7. IMRAN HADDISH AND RIZWAN-UDDIN, "Enhanced Body-Motion Control for Training and Education Using Virtual 3D Models of NPPs", Trans. ANS, p. 104, (2011) 